\title{
Apathy dimensions in Parkinson's disease
}

Ratko Radakovic $^{1234^{*}}$, Richard Davenport ${ }^{2}$, John M. Starr ${ }^{3} \&$ Sharon Abrahams $^{124}$

${ }^{1}$ Human Cognitive Neuroscience, University of Edinburgh, Edinburgh, UK

${ }^{2}$ Anne Rowling Regenerative Neurology Clinic, University of Edinburgh, Edinburgh, UK

${ }^{3}$ Alzheimer Scotland Dementia Research Centre, University of Edinburgh, Edinburgh, UK

${ }^{4}$ Euan MacDonald Centre for MND Research, University of Edinburgh, Edinburgh, UK

Word Count: 3198

Keywords: apathy; Parkinson's disease; motor symptoms, non-motor symptoms; depression; activities of daily living

\section{Key Points:}

- Lack of motivation for planning, organising and attention (Executive apathy) and self generation of thought or behaviour (Initiation apathy) were observed in PD

- Apathy subtype deficits were found to be related to impairment of activities of daily living in PD

- The Dimensional Apathy Scale (DAS) is a valid and reliable multidimensional apathy measure in PD

\footnotetext{
${ }^{*}$ Correspondence to: R. Radakovic, University of Edinburgh, Department of Psychology, 7 George Square, Edinburgh, UK, EH8 9JZ

Email addresses: r.radakovic@ed.ac.uk, radakovic.ratko@gmail.com Tel: +441316511303
} 


\begin{abstract}
Objective: Apathy is a prominent and disabling symptom in PD and is a multidimensional behaviour, but which dimensions are specifically affected is unclear. Therefore the aim of this preliminary study was to determine the psychometric properties of the Dimensional Apathy Scale (DAS) and explore the multidimensional profile of apathy in PD patients. Methods: 34 PD patients, with 30 of their informants/carers, and 34 healthy controls with, 30 of their informants, completed the DAS, Apathy Evaluation Scale (AES) and the Geriatric Depression Scale- Short form (GDS-15). Motor staging and independent living status were recorded. Results: Comparative group analyses revealed that PD patients were significantly more apathetic on self-rated Executive $(p=.01)$ and Initiation $(p=.03)$ dimensions than controls, where only Executive apathy was significantly higher in ratings of patients' informants/carers compared to controls' informants $(p=.02)$. A third of patients were impaired on at least one apathy dimension. Additionally, patients with apathy tended to have more impaired activities of daily living, while none of the apathy dimensions related to motor disability. Conclusion: Our findings show the DAS is a valid and reliable multidimensional apathy tool for use in PD. PD is characterised by an Executive apathy profile as determined by informants/carers, although patients described both Executive and Initiation apathy. This indicates a lack of motivation for planning, organization and attention and lack of initiation of thoughts or behaviours. Further research is needed to determine the cognitive underpinnings of this emerging apathy profile and the clinical impact in PD.
\end{abstract}




\section{Introduction}

Apathy is an important symptom for patients living with Parkinson's disease (PD) and their carers. It is common in PD, affecting between $30 \%$ to $51 \%$ of patients (den Brok et al., 2015; Dujardin et al., 2007; Kirsch-Darrow et al., 2006; Pedersen et al., 2009) and is associated with decreased quality of life (Benito-León et al., 2012; Laatu et al., 2013) and cognitive impairment (Dujardin et al., 2010; Pluck and Brown, 2002). It is also a symptom that affects carers, and is associated with increased distress and burden in caregivers (Leiknes et al., 2010; Leroi et al., 2012).

Apathy is not a one-dimensional symptom, but is a syndrome (Marin, 1991) comprised of several dimensions, domains or subtypes; some differentiation between components of apathy in PD has been suggested (Kay et al., 2012) and with some scales used to measure numerous sub-factorial domains of apathy, such as the Lille Apathy Rating Scale (LARS) e.g. (Dujardin et al., 2007). The LARS measures four domains of apathy pertaining to action initiation (unproductive in day-to-day life and lessened initiative), intellectual curiosity (lack of novelty seeking, interest and motivation), emotion (emotional blunting of responses and diminished concern) and self-awareness (metacognition and awareness). Levy and Dubois (Levy, 2012; Levy and Dubois, 2006) proposed an emerging triad of neurobiological apathy subtypes, which associate with lack of motivation and goal-directed behaviours. These subtypes are Auto-activation apathy as a lack of activity or initiation of thought or action, Emotional-affective apathy which impacts processing of emotional information observable as emotional blunting or indifference and, finally, Cognitive apathy (or Cognitive inertia) as an inability to expand on plans, organise or manage goals associated with plans. A comprehensive review of multidimensional conceptualization of apathy and associated neuroimaging findings provided support for Levy and Dubois apathy subtyping in PD (Pagonabarraga et al., 2015). Specifically, it suggested the most common subtype impairments would be manifested as Cognitive apathy and Emotional-affective apathy, based on neurobiological pathways affected in PD. However, there has been no scale validated in PD that measures these subtypes directly.

The Dimensional Apathy Scale (DAS: Radakovic and Abrahams, 2014) was specifically designed to measure dimensions of apathy known as Executive (planning, 
attention and organization), Emotional (emotional neutrality/indifference) and Initiation (self-generated behaviours or cognition) apathy, which are akin to Levy and Dubois Cognitive, Emotional-affective and Auto-activation apathy subtypes, respectively. It is the only scale created based on Levy and Dubois' triadic apathy subtypes. It has been validated in amyotrophic lateral sclerosis, quantifying a unique apathetic profile deficit of Initiation apathy and measured multidimensional apathy independent of motor disability (Radakovic et al., 2016), therefore making it suitable for use in PD patients.

The aims of this preliminary study were to validate both the self-rated and informant/carer-rated DAS in a sample of PD patients and explore the profile of multidimensional apathy in PD patients. This study also aimed to determine the relationship of multidimensional apathy with other clinical variables, including motor dysfunction, depression and activities of daily living in PD patients.

\section{Methods}

\section{Participants}

Patients were recruited from a Movement Disorders Clinic, lead by RD, between February 2014 and September 2015. 54 probable idiopathic PD patients and their 54 informants/carers were approached to participate. 5 patients were excluded due to preexisting medical problems, including previous brain injury and other significant illness, and 15 patients did not participate. 34 patients and 30 of their informants/carers (spouses, family members or close friends of patients) participated in the study. No PD patients had dementia. 34 matched age-gender-education healthy controls and 30 of their informants (spouses, family members or close friends of controls) from the University of Edinburgh Volunteer Panel were screened for preexisting medical problems and recruited to participate.

Ethical approval was obtained from the National Health Service (NHS) South East Scotland Research Ethics Committee 02 and the School Philosophy, Psychology and Language Sciences (PPLS), University of Edinburgh Ethical Committee, in accordance with the Declaration of Helsinki. Informed consent was obtained from all participants. 


\section{Measures}

All participants (Patients and their informants/carers, controls and their informants) completed two apathy measures, for psychometric comparison (convergent validity), and one depression measure, to explore the relationship of multidimensional apathy with depression. Informants/carers completed the questionnaires about their observations of the patients and controls.

Multidimensional apathy was assessed using the self and informant/carer versions of the DAS (Radakovic and Abrahams, 2014), which is composed of 3 subscales assessing Executive, Emotional and Initiation apathy subtypes. The minimum score for each subscale is 0 (least apathy) and the maximum 24 (most apathy). The scale was validated in an amyotrophic lateral sclerosis (Radakovic et al., 2016). Impairment on the DAS subscales was calculated on the basis of $\geq 2$ SD above the mean of the control sample. The self and informant version of the AES (Marin et al., 1991) were used as a unidimensional measure of apathy, which produces one composite score, with the minimum score of 18 (least apathy) and maximum score of 72 (most apathy). The AES has been validated in PD (Santangelo et al., 2014) with suggested cutoffs of 39 for the self-rated and 40 for the informant/carer-rated versions, which are based on $\geq 2$ SD above the mean of control sample in (Marin et al., 1991).

Depression was measured by the self and informant versions of the Geriatric Depression Scale-Short form (GDS-15: Brown and Schinka; 2005, Yesavage and Sheikh, 1986) with the minimum score is 0 (not depressed) with the maximum being 15 (most depressed). Clinical cutoff of $>6$ were used for presence of depressive symptoms (Wancata et al., 2006).

Informants completed the Lawton Instrumental Activities of Daily Living (LIADL: Lawton and Brody, 1969) to assess functional independence. Total scores range from 0 (low function, dependent) to 8 (high function, independent). Finally, PD patients were staged using the Hoehn and Yahr scale (HY) (Hoehn and Yahr, 1967). Clinician's notes, observation and HY score were used to define on-off state, in relation to motor symptom fluctuations. 


\section{Statistical analysis}

SPSS 21.0 and R were used for statistical analysis. The Shapiro-Wilk test was used to test normality, which served as a basis for whether parametric and non-parametric statistics were used for subsequent analyses. Independent t-tests, Mann-Whitney U tests and Chi-square tests were used to compare demographics, symptom frequency and clinical variables (apathetic patients vs non apathetic patients). Psychometric properties of the DAS were determined using Holm corrected Pearson's $r$ for convergent validity and divergent validity. Cronbach's Standardized alpha was also used to determine internal consistency reliability. 2 x 3 mixed design ANOVAs were used for Group (Patients/Informants vs Controls/Informants) and DAS subscale (Emotional vs Executive vs Initiation) comparisons, along with post hoc t-tests.

\section{Results}

\section{Demographics}

There were no significant differences between age, sex and years of education of PD patients and healthy controls (Table 1). Most informants/carers were spouses, for both patients and healthy controls. $76.5 \%$ of PD patients were taking dopaminergic medication.

\section{Table 1.}

\section{Psychometrics of the DAS}

\section{Table 2.}

Table 2 shows that DAS subscales correlated positively with the AES, with the selfrated Emotional and Initiation subscales correlating strongly and Executive correlating moderately with the AES, and not correlating significantly with the GDS15. The informant/carer-rated DAS subscales scores were positively correlated with the AES, with Executive and Initiation subscales correlating strongly and the Emotional correlating moderately. The Emotional subscale did not significantly correlate with the GDS-15. A positive correlation was observed between the AES and GDS-15, for both self $(r(32)=.55, p<.001)$ and informant/carer ratings $(r(28)=.74$, $p<.001)$. 
The Cronbach's Standardized alpha value for the self-rated DAS was 0.84 and for the informant/carer-rated DAS was 0.92, which were good and excellent, respectively. With regards to DAS subscales, the Executive $($ self $=0.81$, informant $/$ carer $=0.90)$ and Initiation $($ self $=0.76$, informant/carer $=0.90)$ subscales were good to excellent. The self-rated Emotional subscale was found to be questionable (0.56), while the informant/carer-rated version of the Emotional subscale was found to be more acceptable (0.71).

\section{Apathy and Depression}

\section{Table 3.}

Table 3 shows patients' and controls' (self and informant/carer-rated) scores on apathy and depression measures. Apathy was significantly higher on the informantrated AES and on the self-rated DAS total in PD patients compared with healthy controls. Both informant and self-rated PD patient group depression scores differed significantly from those of controls.

There was no significant correlation between the HY scale and either the DAS Executive, Emotional, Initiation or the total scores for either self or informant/carerrated versions.

\section{Patient vs Control DAS comparison (Self-rated and Informant/carer-rated)}

\section{Figure 1.}

In Figure 1a, the Group (Patient vs Control) and self-rated DAS Subscale comparison showed a significant main effect for DAS subscale $(F(2,132)=46.05, p<.001)$, indicating there was a difference between DAS subscales. There was no significant interaction between DAS subscale and Group in self-rated scores $(p=.10)$, but there was a significant main effect for Group $(F(1,66)=5.70, p=.02)$, where overall patients in self ratings were significantly more impaired than controls on the DAS subscales. Post hoc independent t-tests showed self-rated patients scoring higher than 
controls on the Executive $(t(66)=-2.58, p=.01)$ and Initiation $(t(66)=-2.28, p=.03)$ subscales.

In Figure 1b, the Group (Patients' informants/carers vs Controls' informants) and DAS Subscale comparison informant/carer ratings showed a significant main effect for DAS subscale $(F(2,116)=33.86, p<.001)$. There were no significant main effect for Group ( $p=.07)$ or significant Group and DAS Subscale interaction $(p=.19)$. However, post hoc independent t-tests showed that informant/carer-rated patients scored significantly higher than informant-rated controls on the Executive subscale $(t(58)=-2.34, p=.02)$.

There was no significant difference between self-ratings and informant/carer-ratings on DAS subscales for both PD patients and controls, indicating preserved awareness of apathy.

\section{Subscale Impairment}

On the self-rated DAS showed 38\% ( $\mathrm{N}=13)$ of PD patients were impaired on at least one apathy subscale. Of these, eleven were impaired on just one subscale, of which seven were impaired only on the Executive, three only on the Initiation and one only on the Emotional subscale. One was found to be impaired on two subscales, the Executive with Initiation subscales, and one was globally impaired on all three subscales. In controls only two participants were impaired on the DAS, one on the Executive and one on the Initiation subscale. The frequency of abnormal apathy was significantly higher in patients than healthy controls $(p=0.001)$. No such difference was observed on the self AES between patients $(\mathrm{N}=5$ impaired) and controls $(\mathrm{N}=2$ impaired).

On the informant-rated DAS, 33\% ( $\mathrm{N}=10)$ of patients were impaired on at least one subscale. Of these, four were impaired on only one subscale, three of which were impaired only on the Initiation and one on the Emotional subscale. Five were impaired on two subscales, of those three were impaired on Executive with Initiation and two on Executive with Emotional subscales. Global impairment on all three subscales was observed in one patient. The most common impairment on two subscales was that of Executive with Initiation apathy. In informants of controls only 
three participants scored impaired on the DAS, with one on the Executive, one on the Emotional and one on all subscales. Once more, informant/carer ratings, the frequency of patients impaired on apathy subscales was significantly higher than controls $(p=0.03)$. No such difference was observed on the informant AES between patients $(\mathrm{N}=5$ impaired $)$ and controls $(\mathrm{N}=3$ impaired $)$.

\section{Clinical variable comparison}

\section{Table 4.}

Table 4 shows a comparison of clinical variables based on informant/carer DAS ratings where PD patients were separated into two groups: impaired on one or more $(\geq 1)$ subscales and unimpaired on any subscales of the DAS. The only significant difference was found on the instrumental activities of daily living measure (LIADL), where patients with $\geq 1$ subscale impairment had a lower score than those who were unimpaired.

\section{Discussion}

Prevalence of apathy in these PD patients, as determined by the DAS, was $38 \%$ by self rating and $33 \%$ by informant/carer ratings. This is consistent with previously reported apathy prevalence rates (den Brok et al., 2015; Dujardin et al., 2007; KirschDarrow et al., 2006; Pedersen et al., 2009), demonstrating that the DAS is sensitive to apathy in PD. Furthermore the DAS was able to detected more patients than controls with apathy subtype impairments, unlike the AES where the number of patients and controls with apathy were found to be similar. Most importantly the DAS quantified subtype impairments in relation to the Levy and Dubois triadic subtyping. Overall the psychometric properties of the self-rated and informant/carer-rated DAS in PD patients were found to be positive, with good convergent validity. Emotional apathy (that relating to indifference and neutrality) was consistently not correlated with depression in the self-rated and informant/carer-rated DAS, showing good discriminant validity. 
Specific apathy subtype impairments were observable in self-rated PD patients, where they scored significantly higher on both the Executive and Initiation apathy subscales compared to controls. The informant/carer-rated comparison showed only Executive apathy as higher in PD patient compared to controls' informants' ratings. Therefore, Executive apathy appears to be the most reliable subtype impairment in PD patients. Generally, executive dysfunction is well documented in PD and has been shown to have associations with apathy (Dirnberger and Jahanshahi, 2013). Specifically research has found that apathy, as measured by the AES, in PD patients was associated with impaired performance on letter fluency, the Stroop task, more preservative errors in set shifting, backward digit span, free recall and recognition memory (Pluck and Brown, 2002; Varanese et al., 2011). Furthermore, a study found that planning related executive functioning tasks specifically were strongly associated with apathy (Weintraub et al., 2005). The current findings demonstrated that Executive apathy was particularly affected, associated with a lack of motivation for planning, organization and attention (Radakovic and Abrahams, 2014), which relates well to the above mentioned cognitive and executive dysfunction associated with apathy in PD. Previous research (Dujardin et al., 2007, Dujardin et al., 2014) using the LARS found that in PD the most commonly affected apathy domains were action initiation (unproductive in day-to-day life and lessened initiative) and intellectual curiosity (lack of novelty seeking, interest and motivation), with one study (Drijgers et al., 2010) additionally endorsing the emotional dimension (emotional blunting of responses and diminished concern). However, the LARS was only found to be a robust unidimensional apathy measure (Radakovic et al., 2015) and its' proposed dimensions do not map thematically on to Levy and Dubois subtyping unlike the DAS. Future research should use the DAS to assess apathy subtypes in PD and determine how they associate with cognitive and executive dysfunction.

Initiation apathy was also found to be prominent when patients were compared to controls on the self-rated DAS. The DAS has previously shown that amyotrophic lateral sclerosis is characterised by Initiation apathy (Radakovic et al., 2016), whereas this study indicates that PD is characterised by a combination of Executive and, from only the self-rated perspective, Initiation apathy. It could be considered that the role of the informant in caring for the individual and, in doing so, motivating them throughout daily life and the disease course may impact on their perception of apathy 
(i.e. through providing external cues to help the motivate towards an Initiation process). It has been observed that motivation through external cueing has been effective for certain apathy subtypes (Levy, 2012; Levy and Dubois, 2006), which could therefore make it more difficult for some informants/carers to observe and rate certain aspects of apathy, but research is needed to support this. However, when comparing patient and informant/carer ratings, there were no consistent discrepancies between scoring on any of the DAS subscales. This is indicative of preserved awareness of apathy in PD patients and also observation of apathy by their informant/carers.

Emotional apathy was not found to differ between patients and controls, which is contrary to the predictions of previous research (Pagonabarraga et al., 2015). While there were cases of Emotional apathy in our sample, they seldom occurred in isolation, and were most often found in conjunction with another subtype impairment. This is likely due to the overlapping networks and involvement of dopamine in relation to goal-directed behaviour systems (Levy, 2012; Levy and Dubois, 2006). While dopamine depletion in the mesocorticolimbic system results in emotional under-reactivity (Pagonabarraga et al., 2015), akin to Emotional apathy, it has also been implicated in decision-making and strategy formation (Levy, 2012; Levy and Dubois, 2006), akin to Executive apathy. In PD, previous research has shown that early drug naïve patients display more apathy than those undergoing dopaminergic treatment (Zis et al., 2015) and a recent review provided evidence that behaviour syndromes such as apathy are hypodopaminergic, improving with use of dopamine agonists (Castrioto et al., 2016). However, no research has directly examined apathy subtype associations with this type of treatment in PD, which might help further elucidate the apathy-dopamine relationship.

In relation to functional ability, a notable difference was observed with patients impaired on one or more subscales being found to have more difficulties with instrumental activities of daily living. Apathy has been previously associated with problems with activities of daily living in PD patients (Laatu et al., 2013) In our study PD patients were found to be most commonly impaired on two subscales, which were Executive and Initiation apathy. Motivation in relation to planning, organization and attention in combination with initiation of thoughts or behaviours are all important in 
an individual's ability to function independently. Of note, there appeared to be no significant relation between motor disability (HY stages) and apathy dimensions indicating that apathy may have an independent effect on daily life but more research is needed to explore this further.

Due to the small sample size of patients $(\mathrm{N}=34)$ and informants/carers $(\mathrm{N}=30)$, Cronbach's standardized alpha values should be interpreted with caution and restraint, in relation to internal consistency reliability. Additionally, a general point, this is a small sample study that provides the groundwork for further use of the DAS in PD to detect these specific apathy subtypes. Further large-scale, replication is needed to determine the apathy subtype profile in PD and how it relates to other clinical related variables throughout the disease process. With the availability of diagnostic criteria for apathy (Robert et al., 2009) and its application in relation to validating the AES in PD (Santangelo et al., 2014), there is further remit to explore the application of the DAS in relation to clinically observable apathy.

\section{Conclusions}

To conclude, through this study of multidimensional apathy in PD patients, the selfrated and informant/carer-rated DAS was found to be a valid, reliable and effective instrument for measuring multidimensional apathy. PD seems to be characterised by Executive apathy (a lack of motivation for attention, planning and organization) and, to some extent, Initiation apathy (lack of motivation for self-generation of thoughts or actions), in relation to other disorders such as amyotrophic lateral sclerosis. Both the self-rated and informant/carer-rated DAS were not associated with motor disability in PD patients and there was no impaired awareness in relation to apathy subtypes. Further research should explore how apathy subtypes associate with other cognitive functioning, executive functioning and clinical variables, which will help further uncover the mechanisms of different apathy subtypes.

\section{Acknowledgments}

We would like to thank the patients and controls, and their families, for participating in the study.

\section{Funding}


Ratko Radakovic was supported by a scholarship from the Anne Rowling Regenerative Neurology Clinic, Alzheimer Scotland Dementia Research Centre and the University of Edinburgh.

\section{Conflict of interest}

None declared.

\section{Authors' roles}

Radakovic: substantial contributions to conception and design, acquisition of data, analysis and interpretation of data, drafting the article and revising it critically for important intellectual content.

Davenport: substantial contributions acquisition of data, interpretation of data, revising the article critically for important intellectual content.

Starr: substantial contributions to conception and design, interpretation of data, revising the article critically for important intellectual content.

Abrahams: substantial contributions to conception and design, interpretation of data, revising the article critically for important intellectual content.

All authors contributed and approved the final manuscript.

\section{Sponsor's role}

The funders had no role in the in study design, in the collection, analysis and interpretation of data, in the writing of the report and in the decision to submit the report for publication

\section{References}

Benito-León J, Cubo E, Coronell C. 2012. Impact of apathy on health-related quality of life in recently diagnosed Parkinson's disease: The ANIMO study. Mov Disord 27 : 211-218. 
Brown LM, Schinka, JA. 2005. Development and initial validation of a 15-item informant version of the Geriatric Depression Scale. Int J Geriatr Psychiatry 20 : 911 918.

Castrioto A, Thobois S, Carnicella S, Maillet A, Krack P. 2016. Emotional manifestations of PD: Neurobiological basis. Mov Disord 31 : 1103-1113.

den Brok MG, van Dalen JW, van Gool WA, Moll van Charante, EP, de Bie R, Richard E. 2015. Apathy in Parkinson's disease: A systematic review and meta-analysis. Mov Disord 30 : 759-769.

Dirnberger G, Jahanshahi M. 2013. Executive dysfunction in Parkinson's disease: a review. J Neuropsychol 7 : 193-224.

Drijgers RL, Dujardin K, Reijnders JS, Defebvre L, Leentjens, AF. 2010. Validation of diagnostic criteria for apathy in Parkinson's disease. Parkinsonism Relat Disord 16 :656-660.

Dujardin K, Langlois C, Plomhause L, Carette AS, Delliaux M, Duhamel A., Defebvre L. 2014. Apathy in untreated early-stage Parkinson disease: Relationship with other non-motor symptoms. Mov Disord 29 : 1796-1801.

Dujardin K, Sockeel P, Delliaux M, Destée A, Defebvre L. 2010. Apathy may herald cognitive decline and dementia in Parkinson's disease. Mov Disord 24 : 2391-2397.

Dujardin K, Sockeel P, Devos D, Delliaux M, Krystkowiak P. 2007. Characteristics of apathy in Parkinson's disease. Mov Disord 22 : 778-784.

Hoehn MM, Yahr MD. 1967. Parkinsonism: onset, progression, and mortality. Neurology $17:$ 427-442. 
Kay DB, Kirsch-Darrow L, Zahodne LB, Okun MS, Bowers D. 2012. Dimensions of apathy in Parkinson's disease: exploratory factor analysis of the apathy scale. $J$ Parkinsons Dis 2 : 161-166.

Kirsch-Darrow L, Fernandez HF, Marsiske M, Okun MS, Bowers D. 2006.

Dissociating apathy and depression in Parkinson disease. Neurology 67 : 33-38.

Laatu S, Karrasch M, Martikainen K, Marttila R. 2013. Apathy is associated with activities of daily living ability in Parkinson's disease. Dement Geriatr Cogn Disord 35 : 249-255.

Lawton MP, Brody EM. 1969. Assessment of older people: Self-maintaining and instrumental activities of daily living. Gerontologist 9 : 179-186.

Leiknes I, Tysnes OB, Aarsland D, Larsen, JP. 2010. Caregiver distress associated with neuropsychiatric problems in patients with early Parkinson's disease: the Norwegian ParkWest study. Acta Neurol Scand 122 : 418-424.

Leroi I, Harbishettar V, Andrews M, McDonald K, Byrne EJ, Burns A. 2012. Carer burden in apathy and impulse control disorders in Parkinson's disease. Int J Geriatr Psychiatry 27 : 160-166.

Levy R, Dubois B. 2006. Apathy and the functional anatomy of the prefrontal cortexbasal ganglia circuits. Cereb Cortex 16 : 916-928.

Levy R. 2012. Apathy: a pathology of goal-directed behaviour. A new concept of the clinic and pathophysiology of apathy. Rev Neurol (Paris) 168 : 585-597.

Marin RS, Biedrzycki RC, Firinciogullari S. 1991. Reliability and validity of the Apathy Evaluation Scale. Psychiatry Res 38 : 143-162.

Marin RS. Apathy: a neuropsychiatric syndrome. 1991. J Neuropsychiatry Clin Neurosci 3 : 243-254. 
Pagonabarraga J, Kulisevsky J, Strafella AP, Krack P. 2015. Apathy in Parkinson's disease: clinical features, neural substrates, diagnosis, and treatment. Lancet Neurol $14: 518-531$.

Pedersen KF, Larsen JP, Alves G, Aarsland D. 2009. Prevalence and clinical correlates of apathy in Parkinson's disease: a community-based study. Parkinsonism Relat Disord 15 : 295-299.

Pluck GC, Brown RG. 2002. Apathy in Parkinson's disease. J Neurol Neurosurg Psychiatry 73 : 636-642.

Radakovic R, Abrahams S. 2014. Developing a new apathy measurement scale: Dimensional Apathy Scale. Psychiatry Res 219 : 658-663.

Radakovic R, Harley C, Abrahams S, Starr JM. 2015. A systematic review of the validity and reliability of apathy scales in neurodegenerative. Int Psychogeriatr 27 : 903-923.

Radakovic R, Stephenson L, Colville S, Swingler R, Chandran S, Abrahams S. 2016. Multidimensional apathy in ALS: validation of the Dimensional Apathy Scale. $J$ Neurol Neurosurg Psychiatry 87 : 663-669.

Robert PH, Onyike CU, Leentjens AFG, Dujardin K, Aalten P, Starkstein S, Verhey FRJ, Yassavage J, Clement JP, Drapier D, Bayle F, Benoit M, Boyer P, Lorca PM, Thibaut F, Gauthier S, Grossberg G, Vellas B, Byrne J. 2009. Proposed diagnostic criteria for apathy in Alzheimer's disease and other neuropsychiatric disorders. Eur Psychiatry 24 ; 98-104.

Santangelo G, Barone P, Cuoco S, Ramio S, Pezzella D, Picillo M, Erro R, Moccia M, Pellecchia MT, Amboni M, Franco S, Grossi D, Trojano L, Vitale C. 2014. Apathy in untreated, de novo patients with Parkinson's disease: validation study of Apathy Evaluation Scale. J Neurol 261 : 2319-2328. 
Varanese S, Perfetti B, Ghilardi MF, Di Rocco A. 2011. Apathy, but not depression, reflects inefficient cognitive strategies in Parkinson's disease. PLoS One 6 : e17846. DOI: 10.1371/journal.pone.0017846

Wancata J, Alexandrowicz R, Marquart B, Weiss M, Friedrich F. 2006. The criterion validity of the Geriatric Depression Scale: a systematic review. Acta Psychiatr Scand $114: 398-410$.

Weintraub D, Moberg PJ, Culbertson WC, Duda JE, Katz IR, Stern MB. 2005. Dimensions of executive function in Parkinson's disease. Dement Geriatr Cogn Disord 20 : 140-144.

Yesavage JA, Sheikh JI. 1986. Geriatric Depression Scale (GDS) Recent Evidence and Development of a Shorter Version. Clin Gerontol 5 : 165-173.

Zis P, Martinez-Martin P, Sauerbier A, Rizos A, Sharma JC, Worth PF, Sophia R, Silverdale M, Chaudhuri K 2015. Non-motor symptoms burden in treated and untreated early Parkinson's disease patients: argument for non-motor subtypes. Eur $J$ Neurol 22 : 1145-1150. 
Table 1. Demographics for PD patients $(\mathrm{N}=34)$ and controls $(\mathrm{N}=34)$

\begin{tabular}{llll}
\hline & Patient & Control & $\begin{array}{l}\text { Patient vs Control } \\
p \text {-value }\end{array}$ \\
\hline Gender (M/F) & $15 / 19$ & $15 / 19$ & 1.00 \\
Age (mean, SD) & $68.2(9.2)$ & $66.1(9.2)$ & 0.36 \\
Years of Education (mean, SD) ${ }^{\dagger}$ & $14.4(4.8)$ & $16.5(3.0)$ & 0.08 \\
HY Score (mean, SD) & $2.3(1.1)$ & & \\
LIADL Score (mean, SD) ${ }^{\dagger \dagger}$ & $6.8(1.2)$ & \\
Age of onset (mean, SD) years & $68.2(9.2)$ & & \\
Disease duration (mean, SD) & $4.4(3.3)$ & & \\
years & & & \\
\hline
\end{tabular}

$\mathrm{SD}=$ standard deviation; LIADL=Lawton Instrumental Activities of Daily Living; HY=Hoehn \& Yahr scale

Significant difference highlighted in bold

$\dagger \mathbf{N}=\mathbf{3 2}$

$\dagger \dagger \mathbf{N}=\mathbf{2 8}$

Table 2. Patient self-rated and informant/carer-rated DAS subscale correlations compared to standardised apathy (AES) and depression (GDS15) measures

\begin{tabular}{lll}
\hline Self $(\mathrm{N}=34)$ & AES & GDS-15 \\
\hline DAS Executive subscale & $\mathbf{0 . 5 7 * *}$ & $\mathbf{0 . 5 4} *$ \\
DAS Emotional subscale & $\mathbf{0 . 5 9 * *}$ & 0.46 \\
DAS Initiation subscale & $\mathbf{0 . 7 0 * * *}$ & 0.43 \\
DAS Total & $\mathbf{0 . 8 0 * * *}$ & $\mathbf{0 . 6 1 * *}$ \\
\hline Informant/Carer $(\mathrm{N}=30)$ & AES & GDS-15 \\
\hline DAS Executive subscale & $\mathbf{0 . 7 8 * * *}$ & $\mathbf{0 . 7 5} * * *$ \\
DAS Emotional subscale & $\mathbf{0 . 6 3 * *}$ & 0.45 \\
DAS Initiation subscale & $\mathbf{0 . 7 9 * * *}$ & $\mathbf{0 . 7 3} * * *$ \\
DAS Total & $\mathbf{0 . 9 0 * * *}$ & $\mathbf{0 . 8 0 * * *}$
\end{tabular}

DAS=Dimensional Apathy Scale; AES=Apathy Evaluation Scale; GDS-15=Geriatric Depression Scale- Short Form $p<.001 * * *, p<.01 * *, p<.05 *$

Significant correlations highlighted in bold 
Table 3. Apathy and depression descriptive data for PD patients $(\mathrm{N}=34)$ and controls $(\mathbf{N}=34)$

\begin{tabular}{rlll}
\hline & Patient & Control & Patient vs Control $p$-value \\
\hline DAS (mean, SD) & & & \\
Self-rated total & $25.8(8.7)$ & $21.2(7.0)$ & $\mathbf{0 . 0 2}$ \\
Informant/Carer-rated total ${ }^{\dagger}$ & $25.1(12.8)$ & $19.7(9.5)$ & 0.07 \\
AES Score (mean, SD) & & & \\
Self-rated & $30.1(6.9)$ & $28.4(5.5)$ & 0.28 \\
Informant-rated ${ }^{\dagger}$ & $31.4(9.1)$ & $27.2(6.6)$ & $\mathbf{0 . 0 4 5}$ \\
GDS-15 (mean, SD) & & & \\
Self-rated & $3.5(2.9)$ & $1.6(1.8)$ & $\mathbf{0 . 0 0 2}$ \\
Informant-rated & $3.5(3.9)$ & $1.8(2.1)$ & $\mathbf{0 . 0 0 1}$
\end{tabular}

SD=standard deviation; DAS=Dimensional Apathy Scale; AES=Apathy Evaluation Scale; GDS15=Geriatric depression scale- Short form

Significant difference highlighted in bold

$\dagger \mathbf{N}=\mathbf{3 0}$

Table 4. Clinical variable comparison of patients' (informant/carer-rated) impaired on $\geq 1$ DAS Subscales $(\mathrm{N}=10)$ to patients unimpaired on all subscales $(\mathrm{N}=\mathbf{2 0})$

\begin{tabular}{llll}
\hline & $\begin{array}{l}\geq 1 \text { Subscale } \\
\text { Impairment }\end{array}$ & Unimpaired & $p$-value \\
& $1.5(0.7)$ & $2.4(1.1)$ & .59 \\
HY (Mean, SD) & $6.1(1.1)^{\dagger}$ & $7.3(1.1)^{\dagger \dagger}$ & .007 \\
DisDL (Mean, SD) & $4.2(3.1)$ & $4.7(3.3)$ & .58 \\
Age of Onset (Mean, SD) & $64.7(13.8)$ & $70.2(5.8)$ & .35 \\
On-Off-No medication state (\%) & & & \\
$\quad$ On (N=13) & $30.8 \%$ & $69.2 \%$ & .75 \\
$\quad$ Off (N=11) & $36.4 \%$ & $63.6 \%$ & \\
$\quad$ No Medication (N=6) & $33.3 \%$ & $66.7 \%$ & \\
\hline
\end{tabular}

LIADL=Lawton Instrumental Activities of Daily Living; HY=Hoehn \& Yahr scale; SD=standard deviation

Significant difference highlighted in bold

$\dagger \mathbf{N}=9 ; \dagger+\mathrm{N}=19$ 
Apathy Dimensions in PD

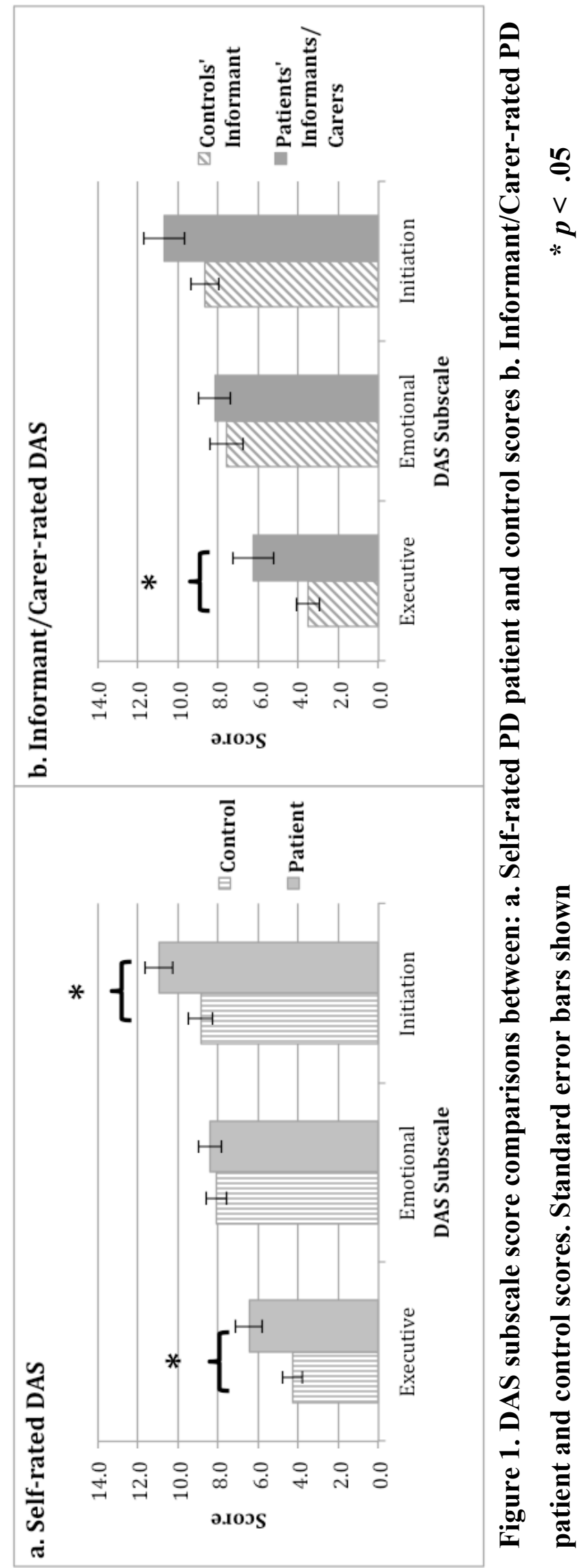


Apathy Dimensions in PD 